\title{
Hyperglycemia is associated with increased length of stay and total cost in patients hospitalized for congestive heart failure
}

\author{
Santhi Adigopula ${ }^{1 *}$, Yan Feng' ${ }^{2}$, Varsha Babu ${ }^{2}$, Konstantinos M. Parperis ${ }^{2}$, \\ Yaw Amoateng-Adjepong ${ }^{2}$, Stuart Zarich ${ }^{1}$ \\ ${ }^{1}$ Division of Cardiology, Department of Internal Medicine, Bridgeport Hospital, Yale University School of Medicine, Bridgeport, USA \\ ${ }^{2}$ Department of Internal Medicine, Bridgeport Hospital, Yale University School of Medicine, Bridgeport, USA \\ Email: ${ }^{*}$ adigopula.santhi@gmail.com
}

Received 18 January 2013; revised 14 March 2013; accepted 17 April 2013

Copyright (C) 2013 Santhi Adigopula et al. This is an open access article distributed under the Creative Commons Attribution License, which permits unrestricted use, distribution, and reproduction in any medium, provided the original work is properly cited.

\begin{abstract}
Objectives: Hyperglycemia is a well-known marker of poor clinical outcomes in acute myocardial infarction and critical illness; however, its effect in congestive heart failure (CHF) is controversial. We hypothesized that persistent hyperglycemia is associated with increased length of stay (LOS) and increased total cost in patients admitted with CHF. Methods: We studied 203 consecutive patients admitted with a primary diagnosis of CHF. Patient characteristics, admission glucose, mean blood glucose (MBG) during the entire hospital stay, length of stay, total cost, and readmission rates were assessed. Persistent hyperglycemia was defined as a MBG level $\geq \mathbf{1 4 0} \mathrm{mg} / \mathrm{dl}$. Results: Patients with persistent hyperglycemia had longer mean LOS (8.1 vs 5.2 days, $p=0.001)$ and higher total hospital costs (median $\$ 8940$ vs $\$ 6892, p=0.01$ ) independent of diabetes status. Similarly, prolonged hospital stay $>7$ days $(38 \%$ vs $21 \% ; p=0.01)$ and total cost $>\$ 10,000 /$ patient $(46 \%$ vs $29 \%$; $p=0.01)$ were seen more commonly in patients with poor glucometrics. Neither admission glucose $>140 \mathrm{mg} / \mathrm{dL}$ or diabetes status was predictive of total costs or LOS. In multivariate linear regression, only $M B G \geq 140 \mathrm{mg} / \mathrm{dl}$ was associated with increased LOS and total cost. Patients with persistent hyperglycemia also had higher 6 months all-cause readmission rates $(51 \%$ vs $37 \%$; p $=0.03$ ). Conclusion: Persistent hyperglycemia (MBG $>140 \mathrm{mg} / \mathrm{dL}$ ), but not admission glucose, was associated with increased LOS, total cost and readmission rates independent of diabetes status. Our study emphasizes the need to further examine the role of glycemic control in patients admitted with $\mathrm{CHF}$.
\end{abstract}

${ }^{*}$ Corresponding author.
Keywords: Congestive Heart Failure; Persistent Hyperglycemia; Length of Stay; Total Cost; Diabetes; Admission Glucose

\section{INTRODUCTION}

Hyperglycemia is associated with adverse clinical outcomes in patients with acute myocardial infarction $[1,2]$, critical illness [3-5], and coronary artery bypass grafting $[6,7]$. Although congestive heart failure (CHF) is by far the most common diagnosis related group requiring acute care hospitalization and is associated with high mortality rates and costs, the prognostic significance of hyperglycemia has not been well established. Similarly, limited data is available regarding the effect of hyperglycemia on costs or length of stay in CHF.

Prior data on hyperglycemia in CHF has focused predominantly on admission glucose levels [8-10]. Persistent hyperglycemia during hospitalization, however, appears to be a better predictor of CV outcomes and mortality when compared to admission glucose in the setting of acute myocardial infarction [2]. The aim of our present study was to examine the role of admission glucose and persistent hyperglycemia during hospitalization on length of stay (LOS) and total cost in patients admitted with a primary diagnosis of $\mathrm{CHF}$, regardless of diabetes status. We hypothesized that persistent hyperglycemia is associated with increased with increased LOS and cost.

\section{METHODS}

We retrospectively identified a cohort of 208 consecutive patients hospitalized for dyspnea and discharged with the primary diagnosis of CHF (excluding patients with acute coronary syndromes) based on any one of the six primary 
CHF ICD-9 codes (428.0, 428.1, 428.2, 428.3, 428.4, 428.9) from January through December 2008 at our university-affiliated community hospital. Detailed information on cost and length of stay, as well as readmission rates, was obtained from the information services department at our hospital after informed consent was obtained from our Institutional Review Board.

This work was previously presented at the American Heart Association scientific sessions [11].

Symptomatic patients with dyspnea were evaluated clinically, as well as biochemically and radiographically for evidence of $\mathrm{CHF}$ requiring admission. After extensive chart/radiographic review of each subject, five patients were excluded for an inability to confirm the diagnosis of $\mathrm{CHF}$ according to the Framingham criteria [12].

\subsection{Data Source}

Electronic medical records were reviewed to abstract demographic data, clinical information, including information on co-morbidities (diabetes, hypertension, coronary artery disease (CAD), chronic renal impairment), NT-Pro-BNP (N-terminal pro-brain natriuretic peptide) levels, estimated glomerular filtration rate according to the MDRD formula (eGFR, $\mathrm{ml} / \mathrm{min}$ ), admission glucose levels, mean blood glucose (MBG) during the entire hospitalization (including all venous and finger stick blood glucose measurements), length of stay (LOS), and total cost. Readmission to our hospital within 6 months following the index admission was also obtained. MBG was calculated by averaging all of the glucose values during entire hospitalization. Based on prior literature [2], patients were divided in to two groups according to their MBG values: persistent hyperglycemia group (MBG $\geq$ $140 \mathrm{mg} / \mathrm{dL}$ ) and controls (MBG $<140 \mathrm{mg} / \mathrm{dL}$ ). A second cut-point of MBG $>200 \mathrm{mg} / \mathrm{dl}$ was examined separately.

\subsection{Outcomes}

The primary outcomes were length of stay and total cost. The secondary outcome was all-cause hospital readmission rate at six months following the index admission.

\subsection{Statistical Analysis}

Analysis was performed using SAS V8 software. Categorical variables, described as proportions, were compared with chi-square tests. Continuous variables were reported as medians with the inter-quartile ranges when non-normally distributed. Non-parametric variables were compared with the Wilcoxon rank sum tests. Multivariate linear and logistic regression analyses were performed to test the association between outcomes with hyperglycemia, after adjustment for effect modifiers, including
NT-Pro-BNP (log (e)), eGFR $<60 \mathrm{ml} / \mathrm{min}$, advanced age ( $\geq 75$ years) and race. A two tailed P-value of $<0.05$ was considered statistically significant. 95\% two-sided confidence intervals were calculated using the exact binomial method.

\section{RESULTS}

A total of 203 patients were included in the analyses. Demographics are shown in Table 1. The mean age of the cohort was $74 \pm 14$ with females comprising $44 \%$ of the total cohort. The mean blood glucose (MBG) for the entire cohort was $137 \pm 48 \mathrm{mg} / \mathrm{dl}$. MBG $\geq 140 \mathrm{mg} / \mathrm{dl}$ (persistent hyperglycemia) was seen in $39 \%$. Forty nine percent of subjects were diabetic, with the majority of diabetics $(70 \%)$ residing in the persistent hyperglycemia group.

In univariate analysis diabetic status, Hispanic race and patients estimated eGFR $<60 \mathrm{ml} / \mathrm{min}$ were the only factors associated with persistent hyperglycemia ( $p<$ 0.01 for both). Age, gender, $\mathrm{EF}<45 \%$, presence of CAD and hypertension were not associated with persistent hyperglycemia. Mean NT-Pro-BNP levels was also similar between the two groups suggesting that the severity of disease was not a major factor in the development of persistent hyperglycemia.

The median cost for the entire cohort was $\$ 7450$ (range: $\$ 1205$ to $\$ 418,815$, inter-quartile range $25^{\text {th }}-75^{\text {th }}$ : $\$ 4979$ - \$12,779). The persistent hyperglycemic group had significantly higher total cost (median $\$ 8940$ vs $\$ 6892, p=0.01)$ than controls. Similarly, the mean total cost was greater in the persistent hyperglycemia group $(\$ 21,924$ vs $\$ 9613 ; p=0.01)$. Using an arbitrary cut off of $\$ 10,000$ to define excessive cost, persistent hyperglycemia was associated with increased incidence of excessive cost (46\% vs 29\%, OR: 2.0: CI 1.2 - 3.7). Only $\mathrm{MBG} \geq 140 \mathrm{mg} / \mathrm{dl}$ and $\mathrm{eGFR}<60 \mathrm{ml} / \mathrm{min}$ were associated with total cost in univariate analysis. In multivariate linear regression, only $\mathrm{MBG} \geq 140 \mathrm{mg} / \mathrm{dl}$ was associated with increased total cost after adjustment for NT-Pro-BNP, renal insufficiency, advanced age and Hispanic ethnicity (Table 2). A graded response of hyperglycemia to total cost was seen when MBG was stratified into 3 sub-groups $(<140,140-199$, and $\geq 200)$ (Figure 1).

The length of stay in the total cohort varied from 1 to 67 days, with median of 5 days (inter-quartile range $25^{\text {th }}$ - $75^{\text {th }}: 3$ - 8 days). As compared to those with MBG < $140 \mathrm{mg} / \mathrm{dl}$, patients with persistent hyperglycemia had higher mean (8.1 vs 5.2 days; $p<0.001)$ and median LOS ( 5 vs 4 days, $\mathrm{p}=0.01$ ). Using an arbitrary cutoff of 7 days as excessive LOS, the persistent hyperglycemia group was associated with an increased incidence of excessive LOS $>7$ days when compared to controls (OR 
Table 1. Demographics and clinical characteristics of the study cohort.

\begin{tabular}{|c|c|c|c|c|}
\hline & $\begin{array}{c}\text { Total cohort } \\
(\mathrm{N}=\mathbf{2 0 3})\end{array}$ & $\begin{array}{c}\text { Hyperglycemic group } \\
(\mathrm{MBG} \geq \mathbf{1 4 0} \mathrm{mg} / \mathrm{DI})(\mathrm{N}=\mathbf{8 0})\end{array}$ & $\begin{array}{c}\text { Control group } \\
(\mathrm{MBG}<140 \mathrm{mg} / \mathrm{dL})(\mathrm{N}=123)\end{array}$ & p Value \\
\hline Age (mean $\pm \mathrm{SD}$, year) & $74 \pm 15$ & $74 \pm 12$ & $74 \pm 16$ & 0.1 \\
\hline Gender & & & & 0.3 \\
\hline Male & $114(56 \%)$ & $41(51 \%)$ & $73(59 \%)$ & \\
\hline Female & $89(44 \%)$ & $39(49 \%)$ & $50(41 \%)$ & \\
\hline Race & & & & $<0.01$ \\
\hline White & 129 & $51(54 \%)$ & $78(63 \%)$ & \\
\hline Hispanic & 31 & $20(25 \%)$ & $11(9 \%)$ & \\
\hline Black & 40 & $7(9 \%)$ & $33(27 \%)$ & \\
\hline Others & 3 & $2(2 \%)$ & $1(1 \%))$ & \\
\hline $\mathrm{DM}$ & 100 & $70(88 \%)$ & $30(24 \%)$ & $<0.01$ \\
\hline$\%$ on insulin & 47 & $38(54 \%)$ & $9(30 \%)$ & \\
\hline CAD & 107 & $45(56 \%)$ & $62(50 \%)$ & 0.4 \\
\hline MedianNT-Pro-BNP $\left(25^{\text {th }}-75^{\text {th }}\right)$ & $7007(3156-14,152)$ & $7056(3004-16,097)$ & $6990(3218-11,874)$ & 0.9 \\
\hline $\mathrm{EF}<45 \%$ & 102 & $40(50 \%)$ & $62(50 \%)$ & 0.8 \\
\hline $\mathrm{GFR}<60 \mathrm{ml} / \mathrm{min}$ & 126 & $61(76 \%)$ & $65(53 \%)$ & $<0.01$ \\
\hline HTN & 179 & $67(84 \%)$ & $112(91 \%)$ & 0.1 \\
\hline Mean glucose mg/dL $($ mean $\pm \mathrm{SD})$ & $137 \pm 48$ & $185 \pm 41$ & $106 \pm 17$ & $<0.01$ \\
\hline Admission glucose $\mathrm{mg} / \mathrm{dl}($ mean $\pm \mathrm{SD})$ & $136 \pm 67$ & $173 \pm 89$ & $112 \pm 29$ & $<0.01$ \\
\hline Discharge glucose $\mathrm{mg} / \mathrm{dL}($ mean $\pm \mathrm{SD})$ & $138 \pm 48$ & $173 \pm 45$ & $109 \pm 25$ & $<0.01$ \\
\hline
\end{tabular}

Table 2. Multivariable linear regression for total cost.

\begin{tabular}{lcc}
\hline Variable & Coefficient & $\mathrm{p} \mathrm{Value}$ \\
\hline $\mathrm{MBG} \geq 140 \mathrm{mg} / \mathrm{dl}$ & 12.5 & 0.03 \\
Pro-BNP $(\log (\mathrm{e}))$ & 2.1 & 0.3 \\
$\mathrm{eGFR}<60 \mathrm{ml} / \mathrm{min}$ & 3.3 & 0.6 \\
Age $\geq 75$ & 5.6 & 0.3 \\
Hispanic & -3.3 & 0.7 \\
\hline
\end{tabular}

2.2: CI $1.1-4.2 ; \mathrm{p}=0.01)$. Only $\mathrm{MBG} \geq 140 \mathrm{mg} / \mathrm{dl}$ and eGFR $<60 \mathrm{ml} / \mathrm{min}$ were associated with LOS in univariate analysis. In multivariate linear regression, only MBG $\geq 140 \mathrm{mg} / \mathrm{dl}$ was associated with increased LOS after adjustment for NT-Pro-BNP, renal insufficiency, advanced age and Hispanic ethnicity (Table 3). Similarly, there was a graded response of hyperglycemia to increased LOS (Figure 2).

Of note, neither the presence of diabetes or admission glucose levels were associated with total cost or LOS. The readmission rate at six months was also increased in the persistent hyperglycemia group (51\% vs $37 \%$; $\mathrm{p}=$ $0.03)$.

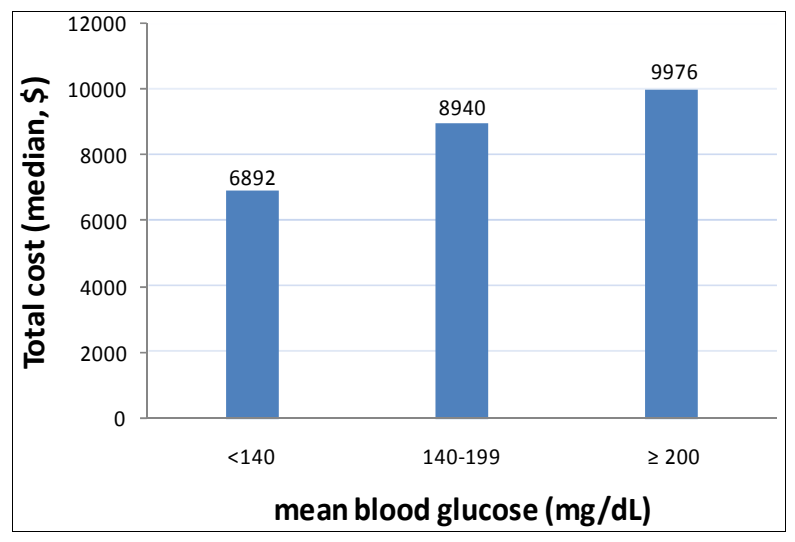

Figure 1. Total cost (median) among different mean blood glucose groups $(\mathrm{p}=0.05)$.

\section{DISCUSSION}

Our study found an association between persistent hyperglycemia and increased total cost and LOS, which was independent of diabetes status and admission glucose levels. Age, median NT-Pro-BNP and left ventricular ejection were similar between the groups suggesting that increased glucose levels were not merely related to 
Table 3. Multivariable linear regression for length of stay.

\begin{tabular}{ccc}
\hline Variable & Coefficient & $\mathrm{p} \mathrm{Value}$ \\
\hline $\mathrm{MBG} \geq 140 \mathrm{mg} / \mathrm{dl}$ & 2.2 & 0.01 \\
Pro-BNP $(\log (\mathrm{e}))$ & 0.6 & 0.5 \\
$\mathrm{eGFR}<60 \mathrm{ml} / \mathrm{min}$ & 0.5 & 0.09 \\
Age $\geq 75$ & 0.9 & 0.2 \\
Hispanic & -0.3 & 0.8 \\
\hline
\end{tabular}

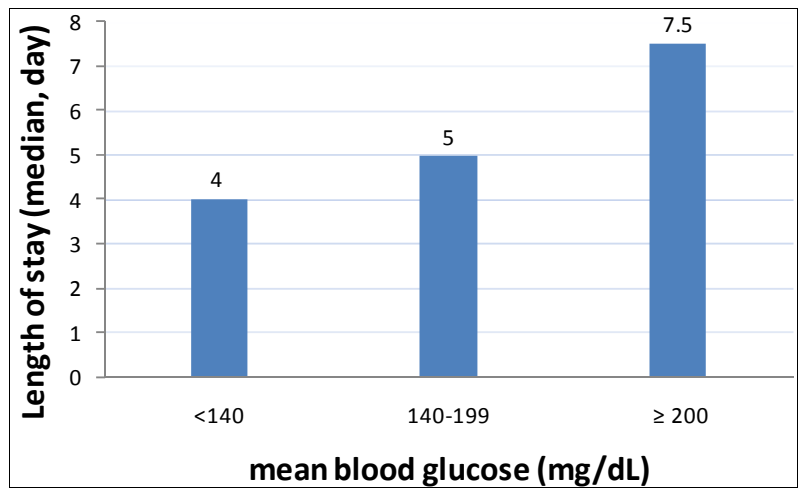

Figure 2. Length of hospital stay (median) among different mean glucose groups $(\mathrm{p}=0.04)$.

the severity of CHF. One may postulate that the observed effects of hyperglycemia on LOS and total cost are likely to be related indirectly to the treatment of complications related to persistent hyperglycemia or directly at therapy aimed at improving diabetic control. Unfortunately the precise mechanism(s) can not be ascertained from this study.

The significance of hyperglycemia on morbidity and mortality in CHF remains controversial. Initial data suggested that hyperglycemia was associated with poor CHF outcomes [8-10]. In a large-scale prospective cohort study fasting plasma glucose was an independent predictor of hospitalization for CHF in high-risk subjects [13]. Similarly, blood glucose levels were associated with increased all-cause mortality in patients with CHF after hospitalization in two moderate-scale cohort studies $[9,14]$. However, a recent report, which evaluated a nationally representative cohort of 50,532 elderly patients hospitalized with CHF, found no significant association between admission glucose levels and either 30-day or 1 -year mortality [10]. Prior studies not only yielded contradictory results, in one study only diabetic subjects were included for analysis [15]. The above studies of hyperglycemia and CHF focused primarily on mortality utilizing admission glucose values and did not assess LOS or costs associated with hospitalization for CHF. There is only limited data that focuses on length of stay [16], which is an important aspect of the overall burden of CHF to the healthcare system, but no prior data is available to our knowledge regarding true cost.

In a prospective cohort study of 212 patients with heart failure exacerbation [16] diabetes was associated with prolonged hospital stay in CHF. Within the diabetic subgroup, both admission glucose and in-patient poor glycemic control were associated with increased LOS, but the non-diabetic cohort was not studied. Our study findings differ in showing a significant association between persistent hyperglycemia and LOS which is independent of diabetes status and admission glucose levels. Additionally, we present data on total cost and readmission rates which appear to be independent of markers of disease severity. As hyperglycemia is observed in nearly half of all patients admitted for CHF [10], the role of hyperglycemia, as well as intensive glucose lowering, warrants future study.

Hyperglycemia is known to have deleterious effects on the cardiovascular system. Hyperglycemia impairs coronary micro-circulatory responses to ischemia, reduces the availability of nitric oxide, attenuates the endothelium -dependant vascular responses, and enhances the production of oxygen derived free radicals [15]. CHF is an insulin-resistant state which is frequently exacerbated by an activation of the renin-angiotensin-sympathetic nervous system axis, as well as an increase in counter-regulatory hormones in the decompensated state. Hyperglycemia may, therefore, not simply be related to the severity of illness of CHF.

\section{STUDY LIMITATIONS}

We acknowledge multiple limitations to our study including its retrospective nature, lack of blinding, modest sample size and data limited to a single center. As hyperglycemia itself may have been the reason that physicians kept patients in longer, a causal relationship between hyperglycemia, LOS and cost can not be ascertained. However, as the persistent hyperglycemia and control groups were similar with regard to age, gender, coronary artery disease status, resting ejection fraction and NTPro-BNP levels, hyperglycemia does not appear to be simply a marker of disease severity. As glucose lowering was at the discretion of the physician, no conclusions can be made regarding individual glucose lowering regimens.

In conclusion, persistent hyperglycemia is associated with increased LOS, total cost and 6 month readmission rates, independent of diabetes status or admission blood glucose. Whether persistent hyperglycemia is a marker or cause for increased LOS and total cost can not be definitively ascertained in this study. Treatment targeted to hyperglycemia itself may be responsible for increased LOS and cost. Whether control of hyperglycemia during an admission for CHF will result in improved LOS or 
cost is unknown. Future prospective studies examining glucometrics, as well as the role of glycemic control in reducing healthcare costs in patients with $\mathrm{CHF}$ are warranted.

\section{REFERENCES}

[1] Malmberg, K., Norhammar, A., Wedel, H., et al. (1999) Glycometabolic state at admission: Important risk marker of mortality in conventionally treated patients with diabetes mellitus and acute myocardial infarction: Long-term results from the diabetes and insulin-glucose infusion in acute myocardial infarction (DIGAMI) study. Circulation, 99, 2626-2632. doi:10.1161/01.CIR.99.20.2626

[2] Kosiborod, M., Inzucchi, S.E., Krumholz, H.M., et al. (2008) Glucometrics in patients hospitalized with acute myocardial infarction: Defining the optimal outcomesbased measure of risk. Circulation, 117, 1018-1027. doi:10.1161/CIRCULATIONAHA.107.740498

[3] Sung, J., Bochicchio, G.V., Joshi, M., et al. (2005) Admission hyperglycemia is predictive of outcome in critically ill trauma patients. The Journal of Trauma, 59, 80-83. doi:10.1097/01.TA.0000171452.96585.84

[4] Whitcomb, B.W., Pradhan, E.K., Pittas, A.G., et al. (2005) Impact of admission hyperglycemia on hospital mortality in various intensive care unit populations. Critical Care Medicine, 33, 2772-2777. doi:10.1097/01.CCM.0000189741.44071.25

[5] Krinsley, J.S. (2003) Association between hyperglycemia and increased hospital mortality in a heterogeneous population of critically ill patients. Mayo Clinic, 78, 14711478. doi: $10.4065 / 78.12 .1471$

[6] McAlister, F.A., Man, J., Bistritz, L., et al. (2003) Diabetes and coronary artery bypass surgery. Diabetes Care, $\mathbf{2 6}$, 1518-1524. doi:10.2337/diacare.26.5.1518

[7] Jones, K.W., Cain, A.S., Mitchell, J.H., et al. (2008) Hyperglycemia predicts mortality after CABG: Postoperative hyperglycemia predicts dramatic increases in mortality after coronary artery bypass graft surgery. Journal of Diabetes and Its Complications, 22, 365-370. doi:10.1016/i.jdiacomp.2007.05.006
[8] Barsheshet, A., Garty, M., Grossman, E., et al. (2006) Admission blood glucose level and mortality among hospitalized nondiabetic patients with heart failure. Archives of Internal Medicine, 166, 1613-1619. doi:10.1001/archinte.166.15.1613

[9] Berry, C., Brett, M., Stevenson, K., et al. (2008) Nature and prognostic importance of abnormal glucose tolerance and diabetes in acute heart failure. Heart, 94, 296-304. doi:10.1136/hrt.2006.110999

[10] Kosiborod, M., Inzucchi, S.E., Spertus, J.A., et al. (2009) Elevated admission glucose and mortality in elderly patients hospitalized with heart failure. Circulation, 119, 1899-1907.

doi:10.1161/CIRCULATIONAHA.108.821843

[11] Adigopula, S., Babu, V., Konstantinos, M.P., et al. (2009) Hyperglycemia in heart failure patients is associated with increased length of stay and costs. Circulation, 120, S548.

[12] McKee, P.A., Castelli, W.P., McNamara, P.M., et al. (1971) The natural history of congestive heart failure: The Framingham study. The New England Journal of Medicine, 285, 1441-1446. doi:10.1056/NEJM197112232852601

[13] Held, C., Gerstein, H.C., Yusuf, S., et al. (2007) Glucose levels predict hospitalization for congestive heart failure in patients at high cardiovascular risk. Circulation, 115, 1371-1375. doi:10.1161/CIRCULATIONAHA.106.661405

[14] Newton, J.D. and Squire, I.B. (2006) Glucose and haemoglobin in the assessment of prognosis after first hospitalisation for heart failure. Heart, 92, 1441-1446. doi:10.1136/hrt.2005.080895

[15] Bhatia, V., Wilding, G.E., Dhindsa, G., et al. (2004) Association of poor glycemic control with prolonged hospital stay in patients with diabetes admitted with exacerbation of congestive heart failure. Endocrine Practice, 10, 467-471. doi:10.4158/EP.10.6.467

[16] Gebreegziabher, Y., McCullough, P.A., Bubb, C., et al. (2008) Admission hyperglycemia and length of hospital stay in patients with diabetes and heart failure: A prospective cohort study. Congestive Heart Failure, 14, $117-$ 120. doi:10.1111/j.1751-7133.2008.07569.x 